This is an Accepted Manuscript of an article published by Taylor \& Francis in Philosophical Magazine, Volume 99, 2019 - Issue 23, available online https://doi.org/10.1080/14786435.2019.1648899 


\section{Reaction/crystallization kinetics studied via in-situ XRD: experimental conditions versus methods of kinetic analysis}

Roman Svoboda*

Department of Physical Chemistry, Faculty of Chemical Technology, University of Pardubice, Studentska 573, 53210 Pardubice, Czech Republic.

\section{Abstract}

Theoretically simulated kinetic data were used to evaluate the errors associated with the common issue of evaluating the in-situ non-isothermal X-ray diffraction data, where the complex multistep temperature program (alternating the non-isothermal heating steps with isothermal steps during which the diffraction patterns are collected) is for the purposes of evaluation replaced by a simple non-isothermal heating performed at the reduced/effective heating rate. The kinetic analysis has shown that, in general, best results are provided by the non-linear optimization methods simultaneously evaluating the data-curves obtained for all the different heating rates. For the nucleation-growth (KMJMA) kinetics the distortive influence of the temperature program parameters increases as follows: heating rate during non-isothermal segments $<$ duration of the isothermal segment $<$ temperature interval between the isothermal segments. The non-optimization methods of kinetic analysis (integral isoconversional methods for evaluation of activation energy $\mathrm{E}$ and master plots for determining the appropriate kinetic model) were found to perform inaccurately, with large degree of randomness based on the selection of starting temperature, and are not recommended for evaluation of the in-situ XRD data - the only exception seem to be the differential isoconversional methods that provided accurate E values. Generalization of the present conclusions for all KMJMA processes is suggested and discussed.

Keywords: in-situ XRD, kinetic analysis, crystallization, KMJMA model, theoretical simulations

\footnotetext{
*Corresponding author: Tel.: +420 466037346 E-mail address: roman.svoboda@ upce.cz
} 


\section{Introduction}

The (powder) X-ray diffraction analysis (P)XRD is a standard analytical tool utilized in both academic and industrial spheres to investigate the crystalline structure of solid-state materials. The in-situ (temperature-resolved) XRD analysis brings numerous advantages to the table, such as commercially accessible measurements in, the -260 to $2400{ }^{\circ} \mathrm{C}$ temperature range, simultaneous possibility to regulate pressure and humidity, or direct XRD studies of reactions and phase transformations. ${ }^{1-5}$ Currently, three main solutions to the sample heating/cooling are commercially available - heated furnaces, direct (strip) heaters and gas flow heaters. Heated furnaces provide good temperature homogeneity (associated with accurate temperature determination), the sample can be easily changed and the sample holder can be selected to avoid interaction with the sample; however this type of temperature regulation is not very precise at low temperatures and enables only rather slow heating/cooling rates. On the other hand, direct heaters offer high heating and cooling rates, as well as the widest range of achievable absolute temperatures. Their disadvantages are the worse temperature uniformity (associated with thermal gradients both within and outside of the sample) and the possible interaction between the sample and the heater/holder (in case of the strip heaters). The gas flow heaters, where the sample is placed in a capillary, provide several advantages associated with the sample positioning (possibility to rotate, transmission mode, accurately reproducible sample position) but the thermal side of this solution is significantly worse (unavoidable temperature gradients, inaccurate determination of temperature) compared to the other two options. ${ }^{1,4,5}$ Nonetheless, combined, these three solutions represent a very wide field of possibilities for temperature regulation.

The technological advance of the in-situ temperature stages is, however, not yet paired with a similar progress regarding the detection of XRD diffraction signal. Therefore, the collection of a standard diffraction pattern still requires significant amount of time (not 
considering the readily unavailable sources of extremely intense XRD radiation, such as synchrotrons). This disproportion between the possibility of very fast heating/cooling and inevitably rather slow pattern collection may potentially result in a major issue in case of nonisothermally determined reaction kinetics, the basis for which is an experiment consisting of alternating short isothermal and non-isothermal steps. The non-isothermal steps define the rate at which the temperature changes in-between the isothermal periods during which the XRD patterns are collected. Nowadays, the in-situ XRD kinetic studies of amorphous-tocrystalline or polymorphic transitions belong to the standard approaches of thermo-kinetic research - see e.g. [6 - 15] In most studies the degree of crystallinity/conversion $\alpha$ obtained from in-situ XRD (usually expressed via evolution of the normalized integrated area under the selected characteristic diffraction peak or part of the pattern) is plotted in dependence on temperature and evaluated by the standard methods of kinetic analysis ${ }^{16,17}$ derived for the assumption of simple linear heating program. Even if the true effective heating rate is correctly calculated for the stepwise temperature program, this approach obviously neglects the possible deviations of kinetics due to the isothermal periods.

In the present paper theoretical simulations paired with the consequent kinetic analysis will be used to explore the magnitude of errors associated with the utilization standard methods of kinetic analysis (which assume simple linear heating program) in case of the nonisothermal in-situ XRD measurements employing the multi-step temperature program.

\section{Kinetic simulations}

The data simulations used in the present article were based on the standard derivative kinetic equation $^{18,19}$ :

$$
d \alpha / d t=I \cdot A \cdot e^{-E / R T} \cdot f(\alpha)
$$


where $\alpha$ is the degree of conversion, $t$ is time, $T$ is temperature, $I$ is the integrated area under the kinetic peak, $A$ is the pre-exponential factor and $E$ is the apparent activation energy of the process. The $f(\alpha)$ function is a representation of a kinetic model; in the present article the most common and most often occurring crystallization kinetics - the nucleation growth Kolmogorov-Mirkin-Johnson-Mehl-Avrami (KMJMA) equation ${ }^{20-25}$ - was utilized in the simulations:

$$
f(\alpha)=m(1-\alpha)[-\ln (1-\alpha)]^{1-(1 / m)}
$$

where $m$ is the model kinetic exponent corresponding to the dimensionality of the process. In order to simulate the in-situ XRD measurement the stepwise temperature program was adopted (see the blue line in Fig. 1). Such temperature program has three variables, the temperature step $\Delta \mathrm{T}$ in-between the two isothermal segments, duration $\Delta \mathrm{t}$ of each isothermal segment (time needed for collection of the XRD pattern of sufficient quality), and the heating rate $\mathrm{q}^{+}$applied during the non-isothermal segments. Based on these variables, the effective heating rate can be calculated:

$$
q_{\text {eff }}^{+}=\frac{\Delta T}{\left(\Delta T / q^{+}\right)+\Delta t}
$$

For illustration, the evolution of $\mathrm{q}^{+}$eff with the corresponding variables $\left(\Delta \mathrm{T}, \Delta \mathrm{t}, \mathrm{q}^{+}\right)$is shown in Fig. 2. Note that graphical determination of $\mathrm{q}^{+}$eff is basically a linear fit through the blue stepwise line from Fig. 1.

For the purpose of the present study all the simulations utilized the following set of the kinetic parameters: $\mathrm{E}=150 \mathrm{~kJ} \cdot \mathrm{mol}^{-1}, \quad \mathrm{KMJMA}$ kinetics, $\mathrm{m}=3, \quad \mathrm{I}=1, \quad A=10^{15} \mathrm{~s}^{-1}$, $\mathrm{T}_{0}=124{ }^{\circ} \mathrm{C}$. In Fig. 1 the example of the derivative kinetic signal simulated for the stepwise temperature program with the " $\Delta \mathrm{T}=2{ }^{\circ} \mathrm{C}, \Delta \mathrm{t}=2 \mathrm{~min}, \mathrm{q}^{+}=2{ }^{\circ} \mathrm{C} \cdot \mathrm{min}^{-1}$ " characteristic (red line) is compared with the signal simulated for a simple non-isothermal linear heating at the rate corresponding to the given characteristic $\mathrm{q}^{+}$eff $=0.66667{ }^{\circ} \mathrm{C} \cdot \mathrm{min}^{-1}$ (black dashed line; $\mathrm{q}^{+}$eff calculated according Eq. 3). In addition, the inset shows the integral kinetic signal as would be obtained during the in-situ XRD experiment with this temperature profile. Each point in 
the inset thus corresponds to the degree of conversion $\alpha$ being calculated for the final stage of the corresponding isothermal segment. In order to map the situation for the most typical range of experimental conditions, datasets similar to that depicted in Fig. 2 were created for all combinations of the following options: $\Delta \mathrm{T}=1,2,3$ and $5{ }^{\circ} \mathrm{C} ; \Delta \mathrm{t}=0.5,1,2,3,5$ and $10 \mathrm{~min}$; $\mathrm{q}^{+}=0.5,1,2,5$ and $10^{\circ} \mathrm{C} \cdot \mathrm{min}^{-1}(120$ datasets in total). Utilization of the sets of different heating rates $\mathrm{q}^{+}$then gives opportunity to perform the standard kinetic analysis ${ }^{16}$ for the given combination of the remaining variables $(\Delta \mathrm{T}, \Delta \mathrm{t})$. The source datasets for these calculations are for the borderline conditions $\left(\Delta \mathrm{T}=1\right.$ and $5{ }^{\circ} \mathrm{C} ; \Delta \mathrm{t}=0.5$ and $\left.10 \mathrm{~min}\right)$ depicted in Figs. 3 and 4. The derivative data in Fig. 3 represent the complete signal record such as would be in case of the multi-step temperature program obtained by e.g. differential scanning calorimetry (DSC), differential thermal analysis (DTA) or differential thermogravimetry (DTG). The simulated data-point density is constant with respect to the time axis, i.e. the isothermal steps contain multiple points. As can be seen, larger temperature steps $\Delta \mathrm{T}$ give rise to more significant distortions compared to the longer pattern collection periods $\Delta \mathrm{t}$. Similar conclusion can be derived also from the integral data depicted (for the same example borderline datasets) in Fig. 4. Note that the integral data were calculated in the way the real in-situ XRD patterns would have been potentially collected (with respect to the largest possible distortion), i.e. the respective degrees of conversion $\alpha$ were calculated for the final stage (last data-points) of the corresponding isothermal segment.

\section{Results}

The kinetic data series simulated for the different $\Delta \mathrm{T}+\Delta \mathrm{t}$ combinations (each data series containing 5 datasets simulated for different $\mathrm{q}^{+}$) were evaluated by means of the nowadays state-of-art approach - the non-linear optimization based on the multivariate kinetic analysis $\mathrm{MKA}^{26}$ : 


$$
\begin{aligned}
& R S S=\sum_{j=1}^{n} \sum_{k=F i r s t_{j} i}^{\text {Last }_{j}} w_{j, k}\left(Y \exp _{j, k}-Y c a l_{j, k}\right)^{2} \\
& w_{j}=\frac{1}{\left|[d \alpha / d t]_{\max }\right|_{j}+\left|[d \alpha / d t]_{\min }\right|_{j}}
\end{aligned}
$$

where $R S S$ is the sum of squared residua, $n$ is number of simulated curves, $j$ is index of the given simulated curve, First $t_{j}$ is the index of the first point of the given curve, Last $_{j}$ is the index of the last point of the given curve, $Y \exp _{j, k}$ is the experimental value of the point $k$ of curve $j, \mathrm{Ycal}_{j, k}$ is the calculated value of the point $k$ of curve $j$ and $w_{j}$ is weighting factor for curve $j$. The weighting factors are especially important in case of the derivative kinetic data, where the heat/mass flow is directly proportionate to the applied heating rate and the kinetic $\mathrm{d} \alpha / \mathrm{dt}$ data (and errors) are thus multiplied by $\mathrm{q}^{+}$when treated with respect to the temperature axis. All the fits were performed under the tested simplifying assumption of the data being collected during a simple non-isothermal heating scan with heating rate equal to $\mathrm{q}^{+}$eff.

Starting with the derivative kinetic data series, the results of MKA are depicted in Fig. 5 - note that both weighting factors alternatives ( $w=1$ and $w$ defined by Eq. 5) were explored. Overall, the MKA fits were very good, in most cases identical to the simulation based on the simple (single-step) heating scan at $\mathrm{q}^{+}{ }_{\text {eff }}$ (the dashed curve in Fig. 1). This is mainly due to the derivative data being simulated in full time resolution, when the non-linear optimization accounts for the data-points within the isothermal segments due to MKA being based on the differential form of the standard kinetic equation (Eq. 1). Interestingly, this indicates that the effective heating rate is far more important for the averaged kinetic signal manifestation (the derivative DSC or DTG data in this case) than the actual distribution of the data-points within the multi-step temperature program. In other words, from the kinetic point of view the multi-step data are evenly distributed along the averaged curve corresponding to $\mathrm{q}^{+}{ }_{\text {eff. }}$ Regarding the particular results, in most cases the monitored kinetic characteristics (E, A, I and $\mathrm{m}_{\text {KMJMA }}$ ) were distorted only negligibly by the simplifying replacement of the multi- 
step temperature program by a single heating step. Only in case of the most severely staggered measurements $\left(\Delta \mathrm{T}=5{ }^{\circ} \mathrm{C}, \Delta \mathrm{t}=10 \mathrm{~min}\right)$ significant distortions arose: $\Delta \mathrm{E}=15-$ $20 \mathrm{~kJ} \cdot \mathrm{mol}^{-1}, \Delta \log \left(\mathrm{A} / \mathrm{s}^{-1}\right)=2-2.5$, and $\Delta \mathrm{m}=0.5-0.6$. On the other hand, even in these cases the errors associated with the integrated area under the optimized kinetic signal (corresponding to e.g. the process enthalpy or mass loss) were only $\sim 3 \%$.

Kinetic evaluation of the data simulated as a resemblance of the in-situ XRD measurements (where only last point of each isothermal segment and no points from the nonisothermal segments were taken into account) resulted, as expected, in larger errors of the kinetic parameters - see Fig. 6. Contrary to the first group of datasets (Fig. 5), here each widening of the gap in-between the data-points (either via increased $\Delta \mathrm{T}$ or $\Delta \mathrm{t}$ ) resulted in gradually increasing deviations of the resulting kinetic parameters. In the absolute value the errors do not largely exceed those observed for the most severe case of the derivative datasets - up to $\Delta \mathrm{E}=30 \mathrm{~kJ} \cdot \mathrm{mol}^{-1}, \Delta \log \left(\mathrm{A} / \mathrm{s}^{-1}\right)=3.5$, and $\Delta \mathrm{m}=0.5$. However, significant deviations occur already for the $\left(\Delta \mathrm{T}=2{ }^{\circ} \mathrm{C}, \Delta \mathrm{t}=3 \mathrm{~min}\right),\left(\Delta \mathrm{T}=3{ }^{\circ} \mathrm{C}, \Delta \mathrm{t}=1 \mathrm{~min}\right)$ and $\left(\Delta \mathrm{T}=5^{\circ} \mathrm{C}, \Delta \mathrm{t}=\right.$ $0.5 \mathrm{~min}$ ) combinations, which all could be considered acceptable from the experimental point of view. Also, well recognizable trends in the deviations can be identified based on Fig. 6 . Evaluation of the kinetic parameters from the in-situ XRD data will in case of the KMJMA kinetics always result in overestimated values of $\mathrm{E}$ and $\mathrm{A}$, and underestimated values of $\mathrm{n}$. The materials characteristic $I$ is in case of the integral data basically defined by the extrapolated baselines ( 0 and $100 \%$ ) and thus no errors/distortions of this parameter arise from the changed kinetics itself.

\section{Discussion}

In the present section we will discuss the sources of errors arising from the in-situ XRD measurements (the analysis will be performed on the integral simulated data introduced 
in Figs. 4 and 6), as well as the possible utilization of different methods of kinetic analysis in case of the in-situ XRD data.

\subsection{Error analysis for the integral (in-situ XRD) data}

Apart from the general influence of $\Delta \mathrm{T}$ and $\Delta \mathrm{t}$ (that was discussed in Section 3), the results of the MKA optimization can be also distorted based on the inconsistence of the deviations associated with different $\mathrm{q}^{+}$- one should bear in mind that MKA is based on a simultaneous optimization of all input data-curves. In order to study the sources of errors and deviations arising from the single-step MKA kinetic evaluations applied to the multi-step temperature programs (resembling the non-isothermal in-situ XRD measurements), the analysis was performed on the integral dataset exhibiting the largest deviations, i.e. the $(\Delta \mathrm{T}=$ $\left.5{ }^{\circ} \mathrm{C}, \Delta \mathrm{t}=10 \mathrm{~min}\right)$ depicted in Fig. 4D. The concept of only several simulated curves (obtained for different $\mathrm{q}^{+}$) being always analyzed during the MKA optimization was employed. The particular sets of the simulated curves are listed in Table $1-$ e.g. in the set A only the data-curves obtained for $\mathrm{q}^{+}=1,2,5$ and $10^{\circ} \mathrm{C} \cdot \mathrm{min}^{-1}$ were evaluated via MKA. Looking at the data in Fig. 4D, it is clear that even under these rather extreme measurement conditions it is only the data obtained at highest underlying heating rate $\mathrm{q}^{+}=10^{\circ} \mathrm{C} \cdot \mathrm{min}^{-1}$ that are apparently largely distorted and shifted to lower temperature (thus almost overlapping with the data simulated for $\left.\mathrm{q}^{+}=5^{\circ} \mathrm{C} \cdot \mathrm{min}^{-1}\right)$. This is indeed reflected in Fig. 7A, where omitting this curve (see set D) resulted in a reduced error of the MKA evaluation compared to the standard evaluation of all five $\mathrm{q}^{+}$curves represented by the dashed line - note that the true values used in the simulations were $\mathrm{E}=150 \mathrm{~kJ} \cdot \mathrm{mol}^{-1}$ and $\mathrm{m}=3$. On the other hand, in case when only the data-curves for $\mathrm{q}^{+}=5$ and $10{ }^{\circ} \mathrm{C} \cdot \mathrm{min}^{-1}$ were evaluated (see set C), completely erroneous results were obtained. The evaluation of E appears to be more influenced by the presence of the largely distorted data - all three sets $(\mathrm{D}, \mathrm{E}, \mathrm{F})$ with the 
omitted $\mathrm{q}^{+}=10^{\circ} \mathrm{C} \cdot \mathrm{min}^{-1}$ data-curve show slight improvement over the standard 5-curve evaluation. The evaluation of mKMJMA, on the other hand, worsens with more curves being omitted from the optimization and only the set $\mathrm{D}$ (where the most distorted curve was omitted) showed improvement over the standard 5-curve evaluation. However, considering the absolute values of deviations depicted in Fig. 7A, the distortions arising from the changed shift of the kinetic data with increasing $\mathrm{q}^{+}$has significantly lower effect on the overall error (expressed by the dashed lines) than the base distortion arising from $\Delta \mathrm{T}$ and $\Delta \mathrm{t}$.

\subsection{Alternative kinetic methods for evaluation of derivative data}

Apart from the non-linear optimization methods (such as the MKA), number of other methodologies exist for enumeration of Eq. $1 .^{16}$ The apparent activation energy is in this respect usually determined via isoconversional methods - it was recently shown that for practical purposes all the respective derivative and integral methods perform similarly in case of complex process scenarios. ${ }^{27}$ Thus for the purpose of the present study the evaluation using one differential (Friedman ${ }^{28}$, Eq. 6) and one integral (Kissinger-Akahira-Sunose $=\mathrm{KAS}^{29}$, Eq. 7) method was applied:

$$
\begin{gathered}
\ln \left([d \alpha / d t]_{\alpha}\right)=-\frac{E}{R T_{\alpha}}+\text { const. } \\
\ln \left(\frac{q^{+}}{T_{\alpha}^{2}}\right)=-\frac{E}{R T_{\alpha}}+\text { const. }
\end{gathered}
$$

where $(\mathrm{d} \alpha / \mathrm{d} t)_{\alpha}, T_{\alpha}$ and $E_{\alpha}$ are the conversion rate, temperature and activation energy corresponding to arbitrarily chosen values of conversion $\alpha$. The evaluations were applied to the selected borderline derivative datasets with the following characteristics: $\left(\Delta \mathrm{T}=1^{\circ} \mathrm{C}, \Delta \mathrm{t}=\right.$ $0.5 \min ),\left(\Delta \mathrm{T}=1{ }^{\circ} \mathrm{C}, \Delta \mathrm{t}=10 \mathrm{~min}\right),\left(\Delta \mathrm{T}=5^{\circ} \mathrm{C}, \Delta \mathrm{t}=0.5 \mathrm{~min}\right)$ and $\left(\Delta \mathrm{T}=5{ }^{\circ} \mathrm{C}, \Delta \mathrm{t}=10 \mathrm{~min}\right)$. In addition, one "intermediate" dataset with $\left(\Delta \mathrm{T}=2{ }^{\circ} \mathrm{C}, \Delta \mathrm{t}=2 \mathrm{~min}\right)$ was also evaluated. The differential Friedman method provided in all cases very precise estimates of the true 
activation energy $\mathrm{E}_{\alpha}=150.00 \pm 0.05 \mathrm{~kJ} \cdot \mathrm{mol}^{-1}$ across the whole explored $\alpha$ range $(0.1-0.9)$, which is a consequence of the standard kinetic equation (Eq. 1) being applied in its differential form. On the other hand, the integral KAS method exhibited considerable errors associated with the data complexity - the corresponding E- $\alpha$ dependences are depicted in Fig. 7B. Whereas the datasets with small $\Delta \mathrm{t}$ provided still relatively acceptable estimates, $\Delta \mathrm{t}=$ 10 min resulted in large uncertainties. In case when the isothermal step was implemented numerous times throughout the kinetic transformation $\left(\Delta \mathrm{T}=1{ }^{\circ} \mathrm{C}, \Delta \mathrm{t}=10 \mathrm{~min}\right)$, the estimated E values are completely incorrect. In case of the isothermal step having been manifested only several times during the relevant time period, the estimates are still relatively accurate but their precision greatly decreased - note the large error bars at $\left(\Delta \mathrm{T}=5{ }^{\circ} \mathrm{C}, \Delta \mathrm{t}=10 \mathrm{~min}\right)$.

The non-fitting model-based analysis also often utilizes the master plots ${ }^{30,31}$ based on a simple transformation of the experimental data. For non-isothermal measurements one can write:

$$
\begin{gathered}
z(\alpha)=\Phi \cdot T^{2} \\
y(\alpha)=\Phi \cdot e^{E / R T}
\end{gathered}
$$

Based on the positions of the maxima of these master plot functions the appropriate kinetic model can be determined. The $y(\alpha)$ function can be further utilized for estimating the parameters of the model; the calculation for the KMJMA kinetics is as follows:

$$
m=\frac{1}{1+\ln \left(1-\alpha_{\max , y}\right)}
$$

where $\alpha_{\max , y}$ is the position of the $y(\alpha)$ function maximum (when plotted in dependence on $\alpha$ ). The $\alpha_{\max , z}$ values are for the present data depicted in Fig. 7C. No significant trend in quality can be recognized with respect to the tested $\left(\Delta \mathrm{T}, \Delta \mathrm{t}, \mathrm{q}^{+}\right)$combinations due to the randomness of the deviation of the single data-point (maximum) position - note that the randomness increases with both, $\Delta \mathrm{T}$ and $\Delta \mathrm{t}$. Since only approximately one third of the obtained $\alpha_{\max , z}$ values lies within the tolerance of the KMJMA model correspondence $\left(\alpha_{\max , z}=0.63 \pm 0.03\right)$, 
the master plots evidently suffer from too high errors for the method to be applicable to the presently investigated multistep measurements. Similar conclusion can be derived also based on the mкмJMA values calculated according Eq. 10 and depicted in Fig. 7D. Also here the qualitative outcome of the tested $\left(\Delta \mathrm{T}, \Delta \mathrm{t}, \mathrm{q}^{+}\right)$combinations is completely random and the obtained KMJMA kinetic exponents are far from being utilizable for deriving the information about the dimensionality of the growing crystallites from the derivative multi-step data. Note however, that the $3 \mathrm{D}$ crystal growth (indicated by mKMJMA $_{\text {Kus }}=3$, as used in the present simulations) is most susceptible to producing large errors during the evaluation according Eq. 10; these errors may have been smaller if e.g. m KMJMA $=1$ was used in the simulations.

Note that the errors provided by the integral KAS method are the consequence of Eq. 7 being derived via integration of Eq. 1 under the assumption of the linear heating, which was true for all the older standard integral isoconversional methods (such as e.g. KAS, $\mathrm{OFW}^{32}$, Starink ${ }^{33}$ etc.) - note that all these methods use simple approximations to solve the integral form of Eq. 1, assuming in their derivation $\mathrm{E}$ to be constant with $\alpha$, and resulting in solutions based on linear optimization such as Eq. 7 (similarly flawed would be the utilization of the popular Kissinger equation ${ }^{34}$ ). The modern flexible integration methods (such as e.g. [35] or [36]) avoid this issue. The most advanced flexible integration methods (such as e.g. [36]) utilize non-linear optimization paired with integration over small segments of either time or temperature ( $\mathrm{E}$ is assumed to be constant only in a small $\alpha$ interval, which is by the way the point of the differential Friedman method), which allows application of this methodology to any temperature program (including e.g. cooling or various complex stepwise procedures) with the result of accurately determined E- $\alpha$ and A- $\alpha$ dependences. For further detailed information the reader is advised to read section 3.3 in [16]. The fact that ignoring the underlying assumptions about the linear $\mathrm{q}^{+}$leads to significantly inaccurate kinetic parameters was also demonstrated e.g. in [37-39]. 


\section{Conclusions}

The errors associated with the kinetic analysis being applied to the derivative and integral multistep data were analyzed for the case of replacing the complex temperature program with a single-step heating at $\mathrm{q}^{+}$eff. The analysis has shown that MKA is very well suited for such analyses. In case of the derivative full-scale data the simplification resulted in only negligible errors in all cases (apart from one, rather extreme and unrealistic program with large $\Delta \mathrm{T}$ and $\Delta \mathrm{t}$ ). In case of the integral data, which are directly proportionate to the real insitu XRD data, it was found that low $\Delta \mathrm{T}$ is the most critical parameter for accurate results with $\Delta \mathrm{T}=1{ }^{\circ} \mathrm{C}$ the errors for all kinetic parameters were small/acceptable even at long pattern collection times (up to $\Delta \mathrm{t}=10 \mathrm{~min}$ ). Larger temperature steps already produced significant distortions of the data and, correspondingly, non-negligible errors in the MKA evaluation. It was also shown that the MKA analysis of the in-situ XRD data does not overly suffer from the increasing data distortions occurring with rising $\mathrm{q}^{+}$- the errors were significantly smaller compared to those produced by $\Delta \mathrm{T}$ and $\Delta \mathrm{t}$. Since the shortening of the integration time $\Delta \mathrm{t}$ is limited by the minimum necessary quality of the XRD pattern, $\Delta \mathrm{T}$ remains to be the most crucial parameter for consideration. In addition, several alternative methods of kinetic analysis were tested for the derivative multi-step data. The isoconversional differential Friedman method provided excellent, undistorted results. On the other hand, the isoconversional integral KAS method as well as the application of the master plots performed rather poorly, providing inaccurate and random-based results (the randomness originates from the arbitrary selection of the isothermal segments temperatures).

With regard to the generalization of the results, the present conclusions are applicable for all processes following the KMJMA kinetics, where the isothermal and non-isothermal crystallization kinetics are similar (failure to meet this requirement may produce additional 
errors [40,41] and favor decreasing of $\Delta \mathrm{t}$ with regard to their minimization). The generalization scaling can be based on adjusting $\Delta \mathrm{T}$ with respect to the number of steps occurring throughout the manifestation of the crystallization process (overall position of the data on the temperature axis is irrelevant) - i.e. the processes taking e.g. double the temperature range (at given $\mathrm{q}^{+}$) to fully evolve need all $\Delta \mathrm{T}$ to be twice as high for the results and conclusions to be transferable. On the other hand, the present paper does not consider crystallization kinetics with different asymmetries (other than KMJMA) or complex crystallization process - these topics will be addressed in a concurrent paper.

\section{Acknowledgments}

This work has been supported by the Czech Science Foundation under project No. 1711753 S.

\section{References}

[1] D.D.L. Chung, P.W. DeHaven, H. Arnold, D. Ghosh. X-ray Diffraction at Elevated Temperatures: A Method for In Situ Process Analysis. Wiley, 1993, ISBN: 9780471187264.

[2] B.E. Warren. X-Ray Diffraction - Dover Books on Physics. Dover Publications, 1990, ISBN: 978-0486663173.

[3] C. Suryanarayana, M.G. Norton. X-Ray Diffraction: A Practical Approach - Artech House Telecommunications. Springer, 1998, ISBN: 978-0306457449.

[4] https://www.bruker.com/

[5] https://www.anton-paar.com/

[6] J.D. Rodriguez-Blanco, S. Shaw, L.G. Benning. The kinetics and mechanisms of amorphous calcium carbonate (ACC) crystallization to calcite, via vaterite. Nanoscale 3 (2011) pp. 265-271.

[7] P. Norby. In-situ XRD as a tool to understanding zeolite crystallization. Curr. Opin. Colloid In. 11 (2006) pp. 118-125.

[8] T.R. Jensen, T.K. Nielsen, Y. Filinchuk, J.E. Jorgensen, Y. Cerenius, E.M. Gray, C.J. Webb. Versatile in situ powder X-ray diffraction cells for solid-gas investigations. J. Appl. Crystallogr. 43 (2010) pp. 1456-1463.

[9] S. Adams, K. Hariharan, J. Maier. Interface effect on the silver ion conductivity during the crystallization of $\mathrm{AgI}-\mathrm{Ag}_{2} \mathrm{O}-\mathrm{V}_{2} \mathrm{O}_{5}$ glasses. Solid State Ionics 75 (1995) pp. 193-201. 
[10] A.T.W. Kempen, H. Nitsche, F. Sommer, E.J. Mittemeijer. Crystallization kinetics of amorphous magnesium-rich magnesium-copper and magnesium-nickel alloys. Metal. Mater. Trans. A 33 (2002) pp. 1041-1050.

[11] L. Ding, Y. Zheng. Effect of template concentration and gel dilution on crystallization and particle size of zeolite beta in the absence of alkali cations. Micropor. Mesopor. Mat. 103 (2007) pp. 94-101.

[12] N. Iqbal, N.H. van Dijk, S.E. Offerman, N. Geerlofs, M.P. Moret, L. Katgerman, G.J. Kearley. In situ investigation of the crystallization kinetics and the mechanism of grain refinement in aluminum alloys. Mat. Sci. Eng. A 416 (2006) pp. 18-32.

[13] S. Ueno, A. Minato, J. Yano, K. Sato. Synchrotron radiation X-ray diffraction study of polymorphic crystallization of SOS from liquid phase. J. Cryst. Growth 198-199 (1999) pp. 1326-1329.

[14] L. Bayés-García, T. Calvet, M.A. Cuevas-Diarte, S. Ueno, K. Sato. In situ synchrotron radiation X-ray diffraction study of crystallization kinetics of polymorphs of 1,3dioleoyl-2-palmitoyl glycerol (OPO). CrystEngComm 13 (2011) pp. 3592-3599.

[15] J.R. Salasin, C. Rawn. In-Situ Kinetic Investigation of Calcium Aluminate Formation. Ceramics 1 (2018) pp. 175-197.

[16] S. Vyazovkin, A.K. Burnham, J.M. Criado, L.A. Pérez-Maqueda, C. Popescu, N. Sbirrazzuoli, ICTAC Kinetics Committee recommendations for performing kinetic computations on thermal analysis data, Thermochim Acta. 520 (2011) pp. 1-19

[17] S. Vyazovkin, K. Chrissafis, M.L. Di Lorenzo, N. Koga, M. Pijolat, B. Roduit, N. Sbirrazzuoli, J.J. Sunol. ICTAC Kinetics Committee recommendations for collecting experimental thermal analysis data for kinetic computations. Thermochim Acta 590 (2014) pp. 1-23

[18] Šesták J. Thermophysical Properties of Solids, Their Measurements and Theoretical Analysis. Elsevier: Amsterdam; 1984.

[19] Šesták J. Science of Heat and Thermophysical Studies: A Generalized Approach to Thermal Analysis, Elsevier, Amsterdam, 2005.

[20] W.A. Johnson, K.F. Mehl. Reaction kinetics in processes of nucleation and growth. Trans. Am. Inst. Min. (Metall) Eng. 135 (1939) pp. 416-42.

[21] M. Avrami. Kinetics of phase change I-general theory. J. Chem. Phys. 7 (1939) pp. 1103-12.

[22] M. Avrami. Kinetics of phase change. II-transformation-time relations for random distribution of nuclei. J. Chem. Phys. 7 (1940) pp. 212-24.

[23] M. Avrami. Granulation, phase change, and microstructure - kinetics of phase change III. J. Chem. Phys. 7 (1941) pp. 177-84.

[24] B.V. Stark, I.L. Mirkin, A.N. Romanovskii. Metals and thermal treatment. Mosk. Inst. Stal. 7 (1935) pp. 5-38.

[25] A.N. Kolmogorov. On the statistical theory of metal crystallization. Izv. Akad. Nauk SSSR, Ser. Mater. 3 (1937) pp. 355-359.

[26] J. Opfermann. Kinetic analysis using multivariate non-linear regression. I. basic concepts. J. Therm. Anal. Calorim. 60 (2000) pp. 641-658

[27] G. Luciano, R. Svoboda. Evaluation of apparent activation energy in case of complex kinetic processes - part 1. J. Therm. Anal. Calorim. - submitted.

[28] H.L. Friedman, Kinetics of thermal degradation of char-forming plastics from thermogravimetry. Application to a phenolic plastic, J. Polym. Sci., Part C 6 (1964) pp. 183-195

[29] T. Akahira, T. Sunose, Method of determining activation deterioration constant of electrical insulating materials, Res. Report Chiba Inst. Technol. (Sci. Technol.) 16 (1971) pp. 22-31 
[30] J. Malek, The kinetic-analysis of nonisothermal data, Thermochim. Acta 200 (1992) pp. 257-269.

[31] R. Svoboda, J. Málek. Interpretation of crystallization kinetics results provided by DSC. Thermochim. Acta 526 (2011) pp. 237-251.

[32] J.H. Flynn, L.A. Wall, General treatment of the thermogravimetry of polymers, J. Res. Nat. Bur. Standards, Part A 70 (1966) 4.

[33] M.J. Starink, The determination of activation energy from linear heating rate experiments: a comparison of the accuracy of isoconversion methods, Thermochim. Acta 404 (2003) 163-176

[34] H.E. Kissinger, Reaction kinetics in differential thermal analysis, Anal. Chem. 29 (1957) 1702-1706

[35] S. Vyazovkin, D. Dollimore, Linear and nonlinear procedures in isoconversionalcomputations of the activation energy of thermally induced reactions in solids, J. Chem. Inf. Comp. Sci. 36 (1996) 42-45

[36] S. Vyazovkin. Modification of the integral isoconversional method to account for variation in the activation energy. J. Comput. Chem. 22 (2001) pp. 178-183

[37] S. Vyazovkin. Is the Kissinger equation applicable to the processes that occur on cooling? Macromol. Rapid Commun. 23 (2002) 771-775.

[38] P.E. Sánchez-Jiménez, J.M. Criado, L.A. Pérez-Maqueda. Kissinger kinetic analysis of data obtained under different heating schedules. J. Therm. Anal. Calorim. 94 (2008) pp. 427-432.

[39] S. Vyazovkin. Isoconversional kinetics of polymers: the decade past. Macromol. Rapid Commun. 38 (2017) 1600615.

[40] Y. Ma, B. Rheingans, F. Liu, E.J. Mittemeijer. Isochronal crystallization kinetics of $\mathrm{Fe}_{40} \mathrm{Ni}_{40} \mathrm{~B}_{20}$ amorphous alloy. J. Mater. Sci. 48 (2013) pp. 5596-5606.

[41] B. Rheingans, Y. Ma, F. Liu, E.J. Mittemeijer. Crystallization kinetics of $\mathrm{Fe}_{40} \mathrm{Ni}_{40} \mathrm{~B}_{20}$ amorphous alloy. J. Non-Cryst. Sol. 362 (2013) pp. 222-230.

\section{Table 1}

Sets of data-curves (simulated at different $\mathrm{q}^{+}$) optimized via MKA during the error analysis performed for the $\left(\Delta \mathrm{T}=5^{\circ} \mathrm{C}, \Delta \mathrm{t}=10 \mathrm{~min}\right)$ dataset.

\begin{tabular}{|l|l|}
\hline set & utilized $\mathrm{q}^{+}$ \\
\hline A & $1,2,5,10$ \\
\hline B & $2,5,10$ \\
\hline C & 5,10 \\
\hline D & $0.5,1,2,5$ \\
\hline E & $0.5,1,2$ \\
\hline F & $0.5,1$ \\
\hline G & $0.5,10$ \\
\hline
\end{tabular}




\section{Figure captions}

Fig. 1: Example dataset simulated for the kinetic parameters: $\mathrm{E}=150 \mathrm{~kJ} \cdot \mathrm{mol}^{-1}$, KMJMA kinetics, $\mathrm{m}=3, \mathrm{I}=1, A=10^{15} \mathrm{~s}^{-1}, \Delta \mathrm{T}=2{ }^{\circ} \mathrm{C}, \Delta \mathrm{t}=2 \mathrm{~min}, \mathrm{q}^{+}=2{ }^{\circ} \mathrm{C} \cdot \mathrm{min}^{-1}$. Step-like (blue color; left and bottom axes) line depicts the applied temperature program, solid (red color; right and top axes) line shows the corresponding derivative kinetic response, dashed (black color; right and top axes) line shows the signal simulated for the simple heating scan and the same kinetic parameters. Inset shows the corresponding integral kinetic signal, $\alpha$ was evaluated in agreement with the concept of in-situ XRD, i.e. last values obtained during each isothermal segment were taken.

Fig. 2: Effective heating rates calculated for different combinations of $\Delta \mathrm{T}, \Delta \mathrm{t}$ and $\mathrm{q}^{+}$. Graph A has $\mathrm{q}^{+}=0.5^{\circ} \mathrm{C} \cdot \mathrm{min}^{-1}$; graph $\mathrm{B}$ has $\mathrm{q}^{+}=10^{\circ} \mathrm{C} \cdot \mathrm{min}^{-1}$; graph $\mathrm{C}$ has $\Delta \mathrm{T}=0.5^{\circ} \mathrm{C}$; graph $\mathrm{D}$ has $\Delta \mathrm{T}=10^{\circ} \mathrm{C}$; graph $\mathrm{E}$ has $\Delta \mathrm{t}=0.5 \mathrm{~min}$; graph $\mathrm{F}$ has $\Delta \mathrm{t}=10 \mathrm{~min}$.

Fig. 3: Example (borderline) derivative kinetic datasets simulated for the listed $\Delta \mathrm{T}, \Delta \mathrm{t}$ and $\mathrm{q}^{+}$ conditions and kinetic parameters: $\mathrm{E}=150 \mathrm{~kJ} \cdot \mathrm{mol}^{-1}, \mathrm{KMJMA}$ kinetics, $\mathrm{m}=3, \mathrm{I}=1$, $A=10^{15} \mathrm{~s}^{-1}$.

Fig. 4: Example (borderline) integral kinetic datasets simulated for the listed $\Delta \mathrm{T}, \Delta \mathrm{t}$ and $\mathrm{q}^{+}$ conditions and kinetic parameters: $\mathrm{E}=150 \mathrm{~kJ} \cdot \mathrm{mol}^{-1}, \mathrm{KMJMA}$ kinetics, $\mathrm{m}=3, \mathrm{I}=1$, $A=10^{15} \mathrm{~s}^{-1}$. Values of $\alpha$ were evaluated in agreement with the concept of in-situ XRD. Lines are only a guidance for eyes.

Fig. 5: Kinetic parameters evaluated by MKA from the derivative kinetic datasets corresponding to all tested $(\Delta \mathrm{T}, \Delta \mathrm{t})$ combinations. Each evaluation was performed either with the weighting factor $\mathrm{w}=1$ or with $\mathrm{w}$ defined by Eq. 5 .

Fig. 6: Kinetic parameters evaluated by MKA from the integral kinetic datasets corresponding to all tested $(\Delta \mathrm{T}, \Delta \mathrm{t})$ combinations.

Fig. 7: A) $\mathrm{E}$ (black data) and $\mathrm{m}$ (red data) values obtained by MKA for the $\left(\Delta \mathrm{T}=5^{\circ} \mathrm{C}, \Delta \mathrm{t}=\right.$ $10 \mathrm{~min}$ ) integral kinetic dataset while omitting various different data-curves - see Table 1 for the definitions of sets A-G. Dashed lines indicate the values obtained for the full dataset (containing all data-curves for all five $\mathrm{q}^{+}$).

B) Results of KAS method applied to the selected derivative kinetic datasets indicated in the legend.

C) Values of $\alpha_{\max , z}$ obtained from the master plot analysis for the selected derivative kinetic datasets - indicated in the legend.

D) Values of $m_{K M J M A}$ obtained from the master plot analysis for the selected derivative kinetic datasets - indicated in the legend. 
Fig 1

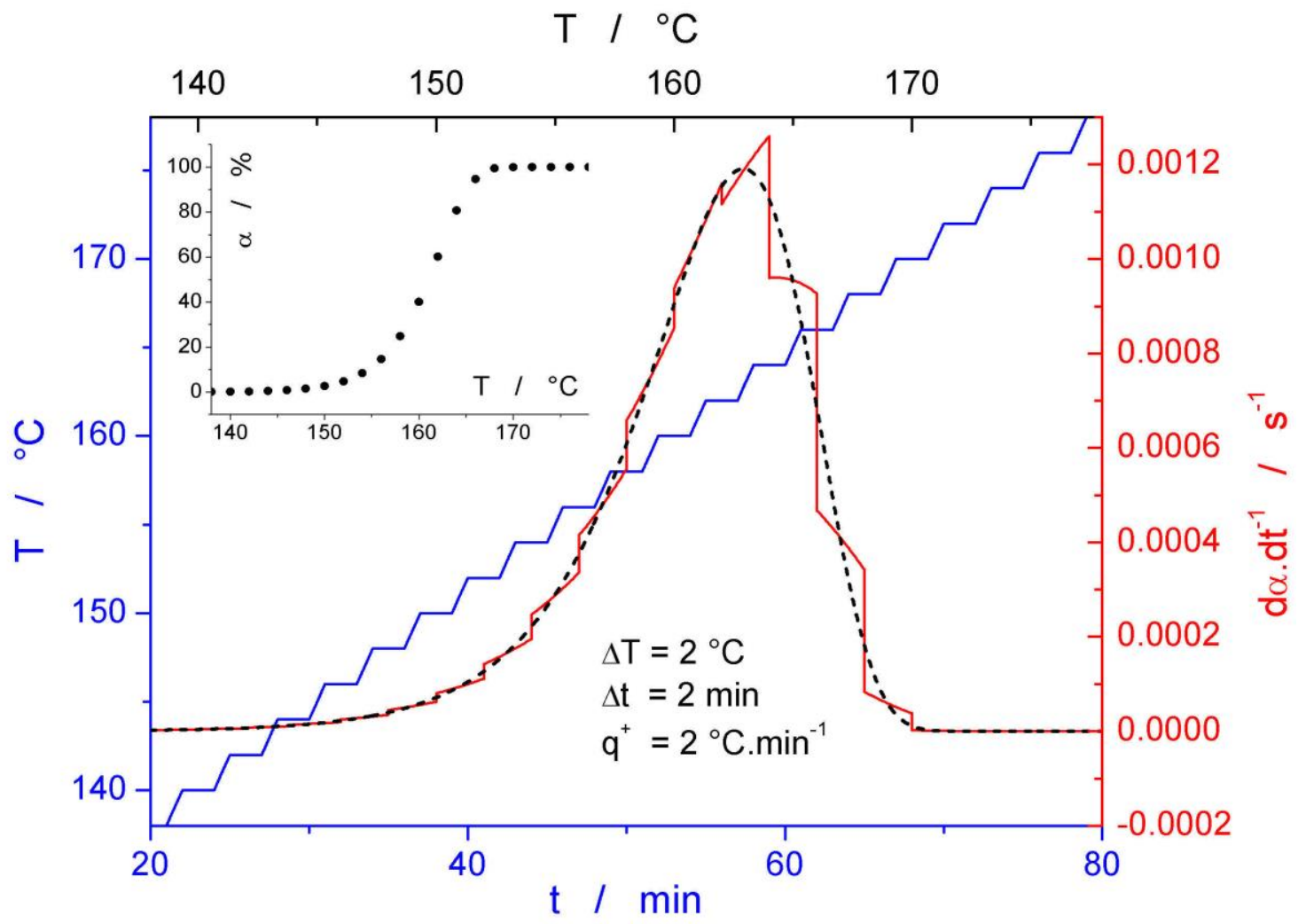


Fig 2
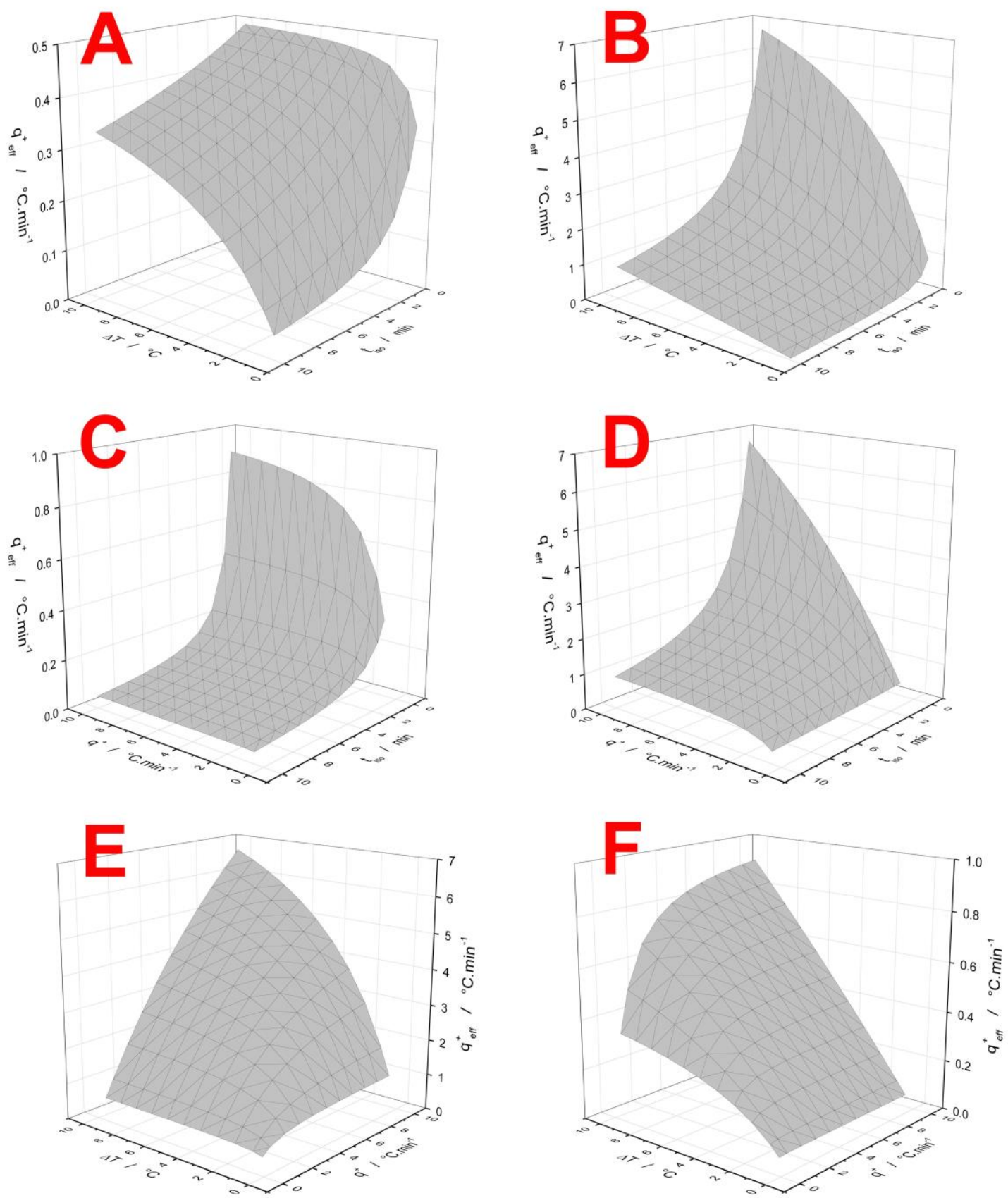
Fig 3
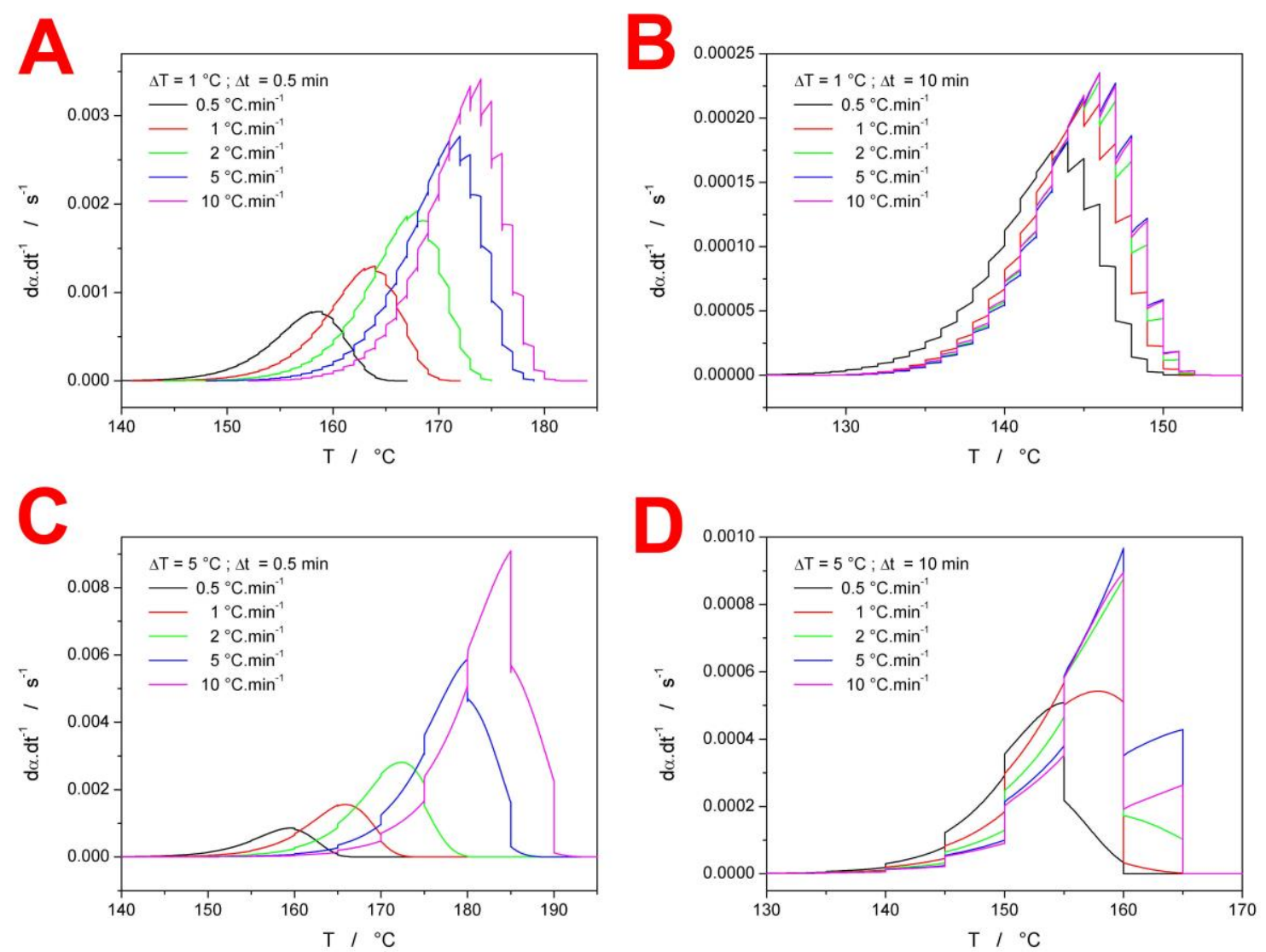
Fig 4
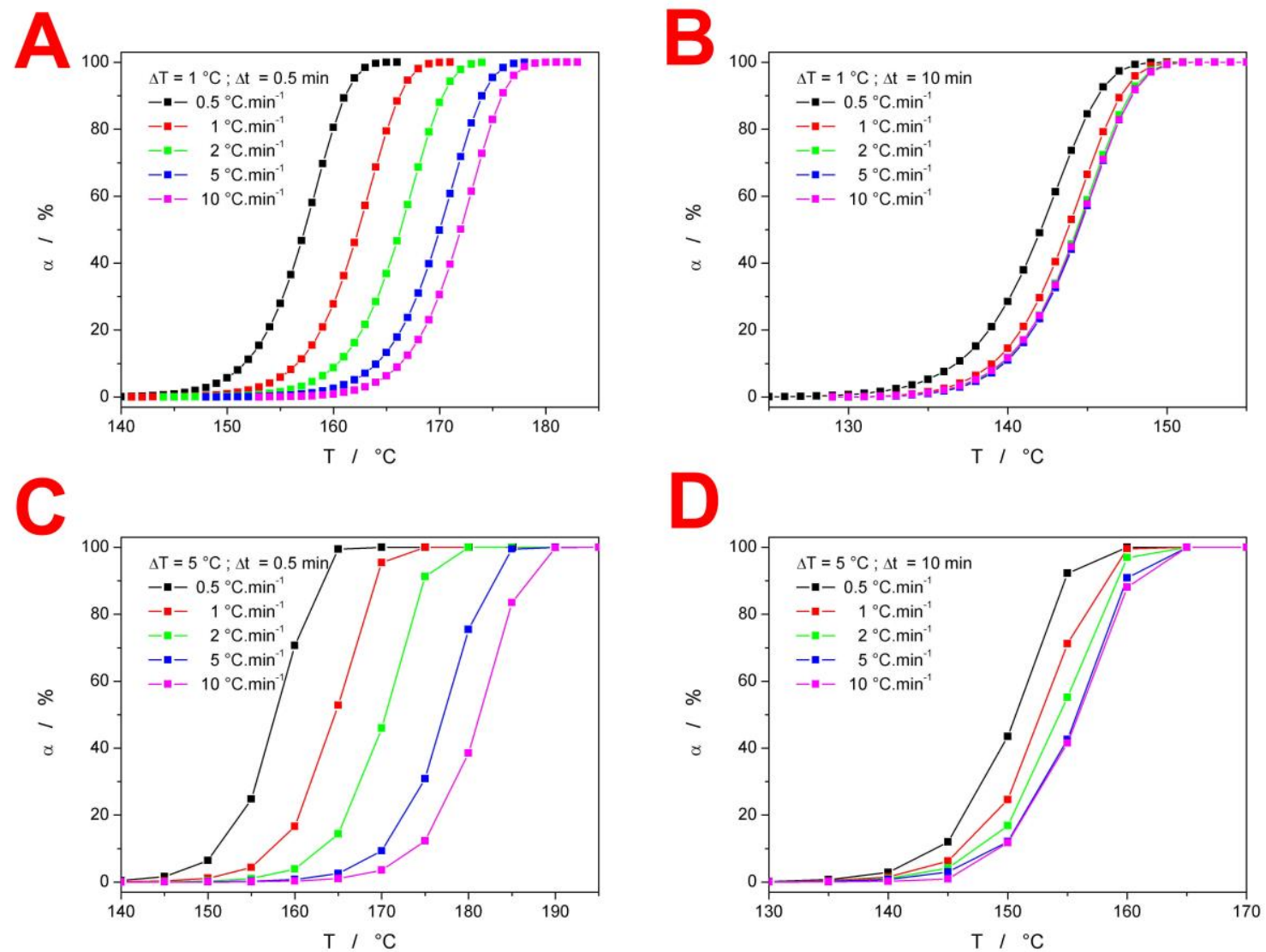
Fig 5
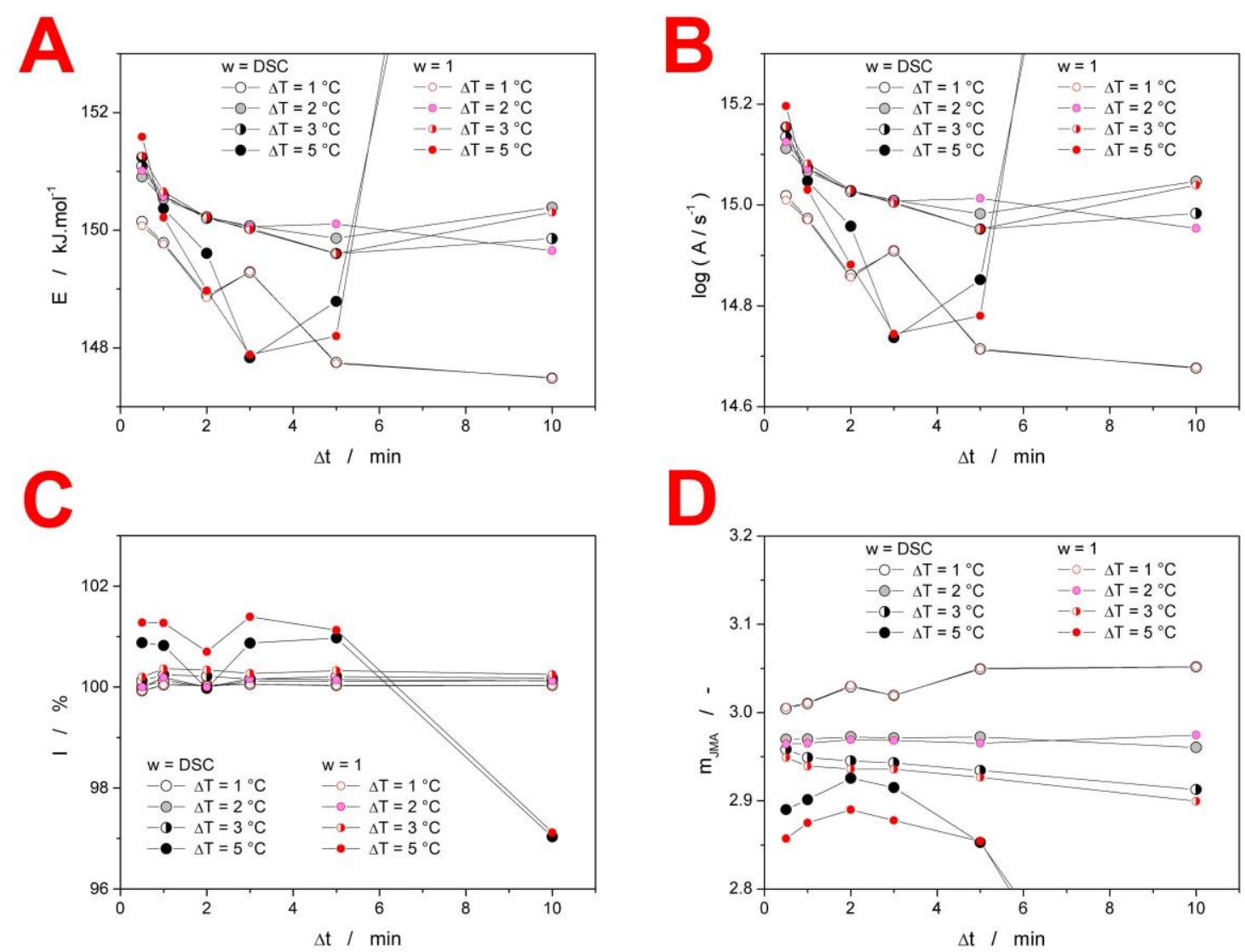
Fig 6
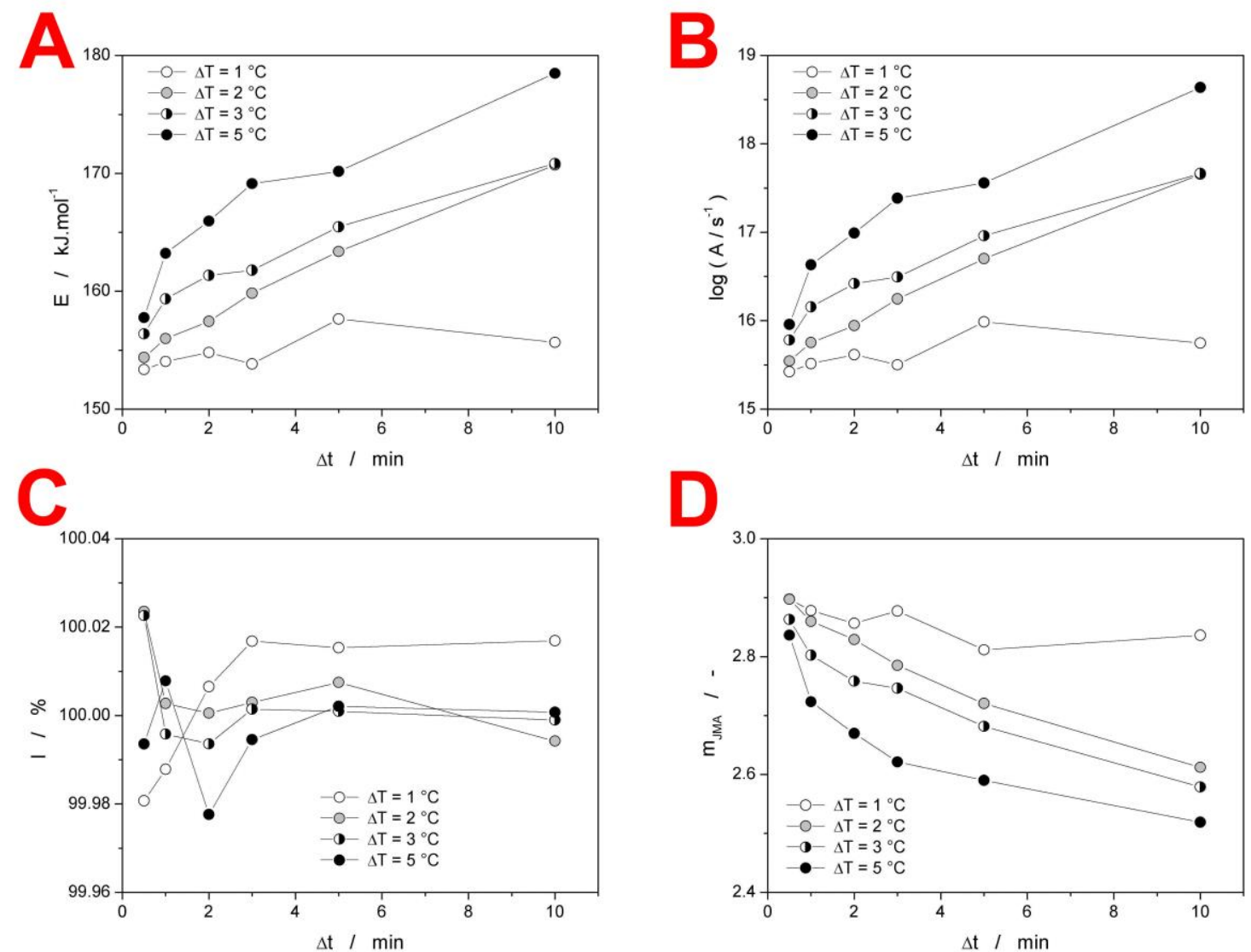

D

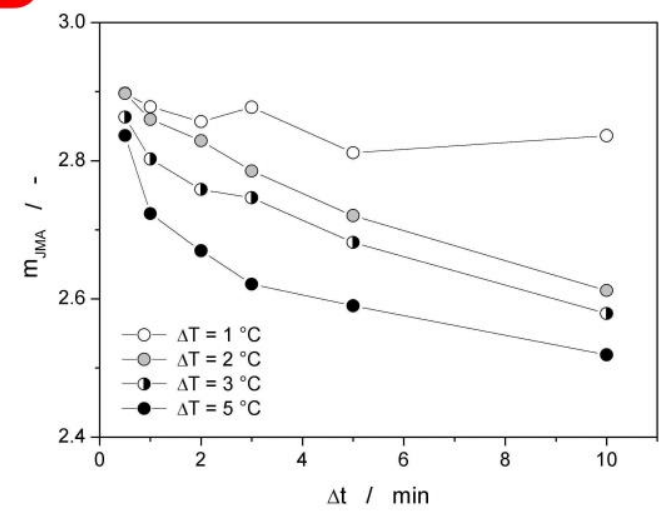


Fig 7
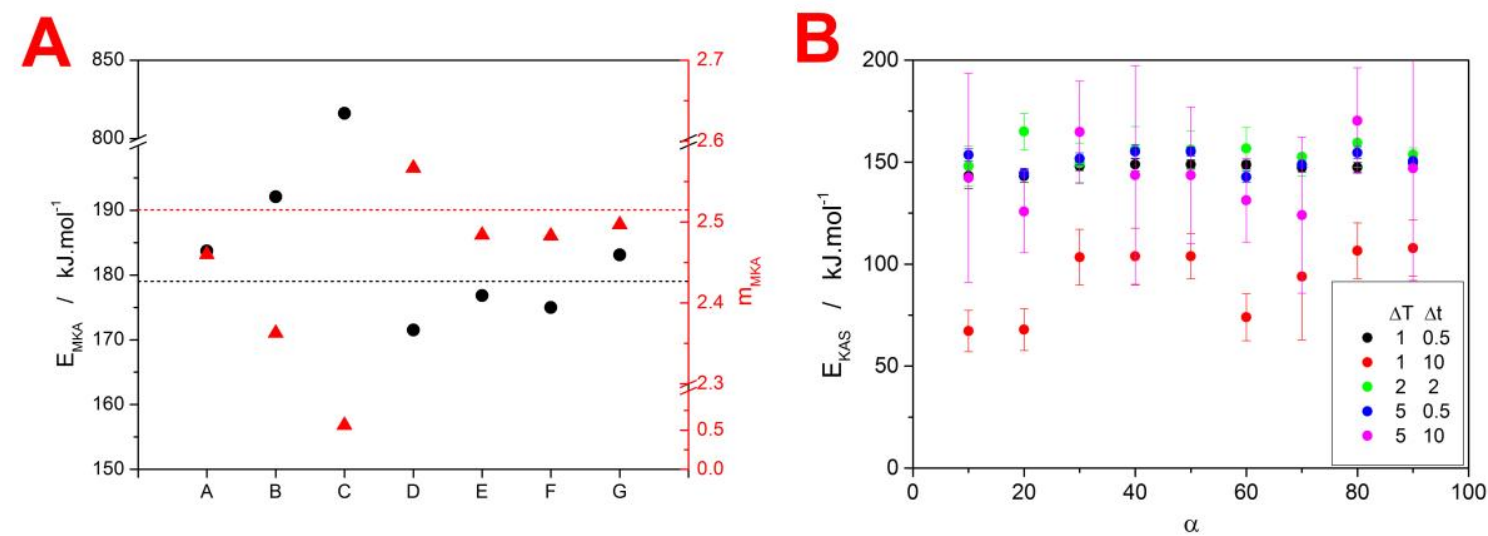

C

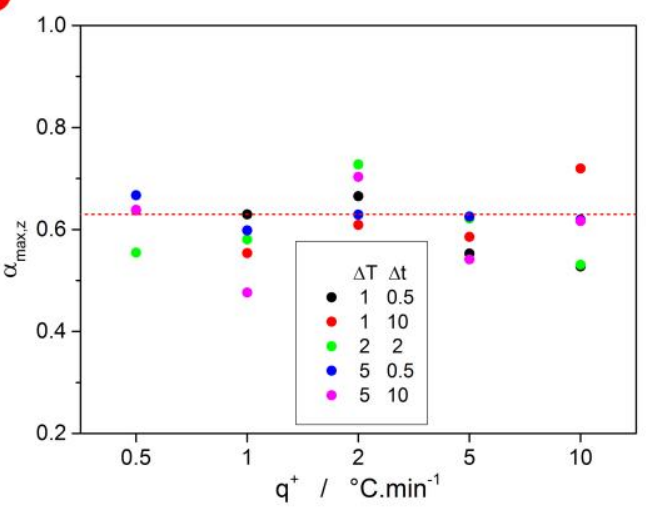

D

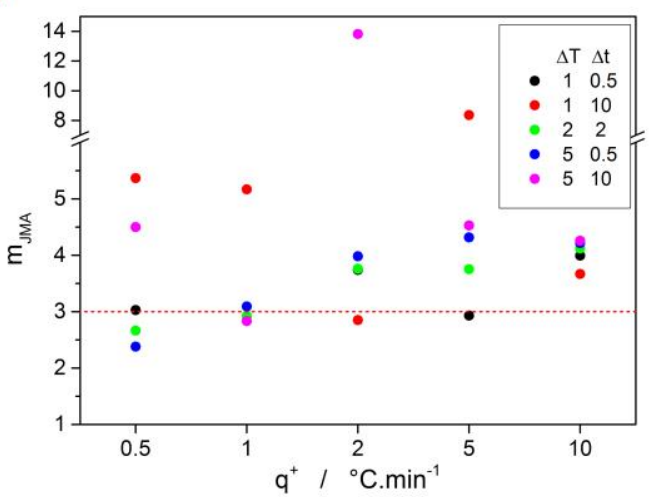

